\section{Prospects for gene therapy of inherited retinal disease}

Division of Molecular Therapy, Institute of Ophthalmology, University College London, London, UK

Correspondence: JWB Bainbridge, Division of Molecular Therapy, Institute of Ophthalmology, University College London, 11-43 Bath Street, London EC1V 9EL, UK Tel: + 440207608 6889; Fax: + 4402076086991 . E-mail: j.bainbridge@ ucl.ac.uk

Received: 1 December 2008 Accepted: 1 December 2008

Published online: 16 January 2009

\begin{abstract}
Gene-based therapies offer the means to address gene defects responsible for inherited retinal disorders. A number of studies in experimental and preclinical models have demonstrated proof-of-principle that gene replacement therapy can mediate significant quantifiable improvements in ocular morphology and visual function. The first results of clinical trials of gene therapy for early-onset severe retinal dystrophy caused by defects in RPE65 show proof-of-concept for efficacy and short-term safety in humans. The challenges for gene therapy of conditions caused by gain-of-abnormal function are being addressed by strategies to knock down expression of the disease allele. Vector-mediated expression of neuroprotective proteins may offer a generic approach for preserving vision in single-gene and multi-gene retinal degenerations. Gene therapy is likely to be most successful where stable expression of the therapeutic transgene can be achieved at an appropriate level in diseases in which retinal development is unaffected and a significant number of target cells survive at the point of intervention. Eye (2009) 23, 1898-1903; doi:10.1038/eye.2008.412; published online 16 January 2009
\end{abstract}

Keywords: retina; gene therapy; vector; gene; RPE65

\section{Introduction}

Inherited retinal degenerations resulting from single-gene defects affect approximately one in every 3000 individuals. ${ }^{1}$ The potential of gene therapy for these conditions has benefited from significant progress in the mapping and cloning of retinal disease genes, of which more than 120 have been identified to date. Significant success has been achieved by gene replacement strategies in models of inherited retinal degenerations caused by loss-of-function mutations in genes encoding proteins that mediate critical functions in photoreceptors or retinal pigment epithelium cells. Strategies for gene therapy of dominantly inherited disease, typically the consequence of gain-of-abnormal function mutations, are also being developed.

The principle of gene replacement therapy is very simple: a gene defect causing lack of function is corrected by providing a normal copy of the gene to the relevant cells. The normal gene is delivered into its target cells using a vehicle or 'vector', which is typically a virus that has been disabled such that it cannot cause spreading of infection by replication. The eye has unique advantages as a target organ for in vivo gene therapy. Its compartmentalized anatomy facilitates accurate local targeting of vector with minimal risk of systemic effects. Intraocular tissues comprise small but stable populations of cells and may be transduced efficiently and stably by small volumes of vector suspension. Immune responses following intraocular administration of vectors are generally attenuated compared with those following systemic administration, and the eye's unique optical transparency enables non-invasive phenotypic investigation of gene expression and therapeutic effects in vivo.

Both viral and non-viral vector systems for gene transfer to ocular tissues have been extensively evaluated. The efficiency and duration of expression of non-viral vectors, which can transduce a wide range of ocular cell types, can be improved considerably by electrotransfer and iontophoresis., ${ }^{2,3}$ Adenoviral vectors efficiently target cells of the outer retina $^{4,5}$ but their duration of expression is limited by immune responses to the vector. ${ }^{6}$ Newer, helper-dependent Ad vectors may mediate longer-term expression. ${ }^{7}$

Recombinant adeno-associated virus (rAAV) vectors offer a range of candidates for retinal 
gene transfer with contrasting tissue tropisms and expression kinetics. $^{8-14}$ In large animal models, rAAV vectors mediate stable expression in the retina, which is maintained for several years. ${ }^{11,15}$ Transgene expression that is sustained in the long term is a highly attractive feature, offering the means to target many life-long retinal disorders after a single administration of vector. The tissue specificity and expression kinetics of rAAV vectors are highly dependent on the vector serotype as well as the anatomical compartment of delivery within the globe. The development of hybrid 'pseudotyped' rAAV vectors, in which an rAAV plasmid is packaged within a capsid derived from AAV of a different serotype, has considerably expanded the available repertoire of AAV vectors. $\mathrm{rAAV}-2 / 2$ (rAAV-2 plasmid packaged in rAAV-2 capsid) mediated expression is typically of delayed onset, gradually increasing in efficiency to reach stable levels of expression after $2-4$ months. In contrast, rAAV-2/1, AAV-2/5, and AAV-5/5 vectors mediate gene expression of rapid onset, with evidence of expression as early as 3-4 days after vector delivery. Although AAV-2/2 and AAV-2/5 transduce both photoreceptors and RPE cells, AAV-2/4 and AAV-4/4 mediate expression that is restricted to the RPE. ${ }^{11} \mathrm{AAV}-5$ pseudotyped vectors mediate successful gene replacement in rodent and large animal models of inherited retinal degenerations. ${ }^{16,17}$ The range of pseudotyped rAAV vectors offers an array of candidates, from which an appropriate vector might be selected according to a desired expression profile. Traditionally considered to be limited by a relatively small packaging capacity, the possibility that the $\mathrm{rAAV} 2 / 5$ capsid can incorporate up to $8.9 \mathrm{~kb}$ of genome greatly expands the therapeutic potential of this vector system to include applications such as Stargardt's disease and Usher's syndrome. ${ }^{18}$

Lentivirus-based vectors are attractive candidates for ocular gene transfer because of their large genomic capacity and their ability to stably transduce non-dividing cell populations. Lentiviral vectors mediate efficient sustained expression in the retinal pigment epithelium, and variable expression in photoreceptors. ${ }^{19-22}$ Lentivirus-mediated transduction of photoreceptor cells is less predictable than transduction of RPE cells, but is reported to occur under certain circumstances depending on retinal maturity, the promoter sequence used, and anatomical barriers. Photoreceptor transduction is evident after subretinal vector delivery in neonatal rodents when driven by a rhodopsin promoter, but the efficiency of photoreceptor transduction in adults is relatively low. ${ }^{23}$ The efficiency of photoreceptor cell transduction in adults can be improved by local retinal trauma and by enzymatic disruption of the inter-photoreceptor matrix, suggesting that anatomical barriers to tissue penetration by vector particles may be a limiting factor. . $^{23,24}$

In addition to efficiency and non-immunogenicity, the ideal choice of vector for a given ocular application is dependent on its genomic capacity, its natural tropisms for the target cells' tissues, and its time courses of expression. As ocular tissues are highly compartmentalized, the pattern of tissue transfection by a given vector is also dependent on its site of intraocular administration. The delivery of rAAV vectors into the subretinal space, for example, results in the transduction of photoreceptors and retinal pigment epithelial cells, whereas injection of the same vector into the vitreous targets only ganglion cells in the inner retina, at least in the fully developed retina. ${ }^{5}$

\section{Gene therapy for photoreceptor-based loss-of-function defects}

Considerable progress has been made in the development of gene replacement therapies for retinal degenerations resulting from gene defects in photoreceptor cells. rAAV-mediated gene replacement of peripherin, a structural protein critical for stability of discs in the photoreceptor outer segment, results in restoration of retinal ultrastructure and function in the rds mouse. ${ }^{25}$ Improved function in this model is not sustained in the long term, however, possibly because the intervention does not slow the rate of progressive photoreceptor apoptosis. ${ }^{26}$ rAAV-mediated gene replacement of retinitis pigmentosa GTPase regulator interacting protein, which is involved in protein trafficking along the photoreceptor-connecting cilium, restores normal protein localization and preserves retinal function. ${ }^{27}$ In a model of $\mathrm{X}$-linked retinoschisis, AAV-mediated expression of retinoschisin in photoreceptors results in structural and functional improvements that are maintained for longer than 1 year. ${ }^{17,28,29}$ In a model of autosomal recessive Stargardt's disease, lentivirus-mediated expression of ABCA4 results in reduced accumulation of $\mathrm{A} 2 \mathrm{E}$ in the retinal pigment epithelium, ${ }^{22}$ despite relatively inefficient transduction photoreceptors, suggesting that Stargardt's disease may be amenable to gene therapy. Finally, in a model of achromatopsia, cone-targeted rAAV-mediated gene replacement of cone-specific alpha transducin subunit (Gnat2) can improve cone function and visual acuity. ${ }^{30}$

\section{Gene therapy for RPE-based loss-of-function defects}

The RPE is particularly amenable to efficient transduction by vectors delivered to the subretinal space by virtue of its monolayer structure and natural phagocytic function. In a model of recessive retinitis pigmentosa, gene replacement of the receptor tyrosine 
kinase MerTK improves the phagocytic defect and preserves retinal function. ${ }^{31,32}$ In a model of ocular albinism, gene replacement of OA1, which is responsible for organizing melanosomes in the RPE, results in improved retinal function as shown by

electroretinography. This suggests that gene therapy may be effective even in conditions in which there is dominant developmental abnormality. ${ }^{33}$ In a model of Usher 1B, lentivirus-mediated expression of the myosin VIIa gene MYO7A can restore the normal apical location of melanosomes in RPE cells, and correct the abnormal accumulation of opsin in the photoreceptor-connecting cilium, suggesting that gene replacement strategy for Usher 1B therapy is feasible.

Defects in the gene RPE65, which encodes a retinal isomerase responsible for regenerating visual pigment after exposure to light, typically cause early-onset severe retinal dystrophy with impaired vision from birth and a progressive degeneration that leads to complete blindness in early adulthood. There is no known treatment, but as retinal structure in this condition is relatively well preserved despite poor function, gene transfer offers the possibility of an improvement in vision that is measurable within the short term. Restoration of visual cycling may be detected non-invasively by increased fundus autofluorescence, which is typically low or absent in affected individuals. ${ }^{34}$ Proof-of-principle that AAV-mediated gene replacement therapy can stably improve retinal function has been demonstrated in the Swedish Briard dog, which is homozygous for a null mutation in RPE65. ${ }^{11,16,35,36}$

The first clinical trial of gene therapy for defect RPE65 began in February 2007 at the UCL Institute of Ophthalmology and Moorfields Eye Hospital London (NCT00643747). Two further trials started later the same year at the Children's Hospital of Philadelphia (NCT00516477) and at the University of Pennsylvania (NCT00481546). Each trial investigated the safety and efficacy of gene replacement therapy in three adults with advanced disease caused by defects in RPE65, and involved subretinal injection of rAAV vector expressing human RPE65. The first results show that the technique is feasible, can be safe in the short term and can lead to improvement in visual function within a period of months, even at a relatively advanced stage of the disease. $^{37-40}$

Subretinal injection of vector was achieved in all subjects. There were no significant intra-operative complications, and all induced detachments resolved spontaneously within $24 \mathrm{~h}$. The vector was delivered to the submacular space in six of the nine subjects. The post-operative development of a macular hole after submacular delivery in one subject was not considered clinically significant because the pre-existing visual acuity was very low, ${ }^{38}$ but would be relevant in subjects with better baseline function and illustrates the risks of surgery involving atrophic retina. Foveal thinning was apparent on ocular coherence tomography in one subject, ${ }^{40}$ but despite temporary retinal detachment visual function recovered to pre-operative levels in all subjects. There were no significant intraocular inflammatory responses to vector injection. The long-term ocular and systemic safety of the procedure will be known only after further follow-up.

All the three groups reported improvements in aspects of visual function. The London group found no change in visual acuity but measured a progressive improvement in retinal sensitivity in one of the three subjects that was statistically significant and associated with an improvement in visually guided mobility in dim light. The Philadelphia group reported improvements in visual acuity and visual fields in all the three subjects and improvement in navigational testing. The Pennsylvania study found no change in visual acuity but measured an increased extent and sensitivity of daylight fields, and locally improved function of both rod and cone photoreceptors that was proportional to the number of surviving photoreceptors estimated by outer retinal thickness on ocular coherence tomography.

Both the US groups reported objective evidence of improved retinal function by pupillometry. Although there was no reported improvement on electroretinography in any of their subjects, this technique is relatively insensitive and even an absent response does not exclude useful visual function. The demonstration of improved retinal responses in these adult subjects by pupillometry, but not by electroretinography, suggests that the extent of the effect of gene therapy at this level of degeneration is relatively modest. The duration for which visual improvement is maintained in these subjects, and the extent to which further retinal degeneration is slowed, will become apparent only after several years.

Although the protocols of the three trials share common features, the differences in study protocol between these trials in terms of inclusion criteria, vector titre and volume, and promoter sequence are expected to yield complementary data that will help to inform the development of optimal vector design and timing of delivery.

Preclinical work suggests that gene replacement therapy for RPE65 is most likely to be effective in affected individuals at an early age as younger subjects have less advanced retinal degeneration. The effect of gene replacement therapy on visual acuity might also be influenced by pre-existing ambylopia resulting from stimulus deprivation during early childhood in affected individuals. It seems likely that the optimal timing of 
intervention will be at a point during the critical sensitive period of visual development. Now that the first phases of the clinical trials have shown the feasibility and short-term safety of subretinal vector delivery in adults with relatively advanced disease, younger subjects with better baseline visual function are being included. This next phase of the trials will help define a window of opportunity for the most favourable timing of intervention.

\section{Gene therapy for gene defects causing gain-of-abnormal function}

The expression of mutant genes causing dominantly inherited retinal degenerations may be suppressed by ribozyme-based strategies or RNA interference. Ribozyme-based strategies specifically cleave mutant mRNAs while sparing the wild-type molecule. The development of an individualized ribozyme for each mutation may not be feasible for heterogenous diseases such as retinitis pigmentosa; over 100 different mutations have been described for the rhodopsin gene alone (www.sph.uth.tmc.edu/RetNet/disease.htm; www.retinainternational.org/sci-news/rhomut.htm). An alternative approach, however, may be to use a non-specific ribozyme that cleaves all the rhodopsin mRNAs (including wild-type as well as mutant rhodopsin mRNAs) by targeting a commonly accessible site, in combination with gene replacement of a wild-type rhodopsin designed to be resistant to the ribozyme. ${ }^{41}$ More recently, the potential of RNA interference (RNAi) for silencing expression of mutant genes has attracted wide interest as an alternative strategy for the treatment of dominantly inherited disorders. ${ }^{42}$ An important challenge for RNAi-based therapy is the development of an effective means of delivering short interfering RNAs to the target cell population. Maintaining therapeutic levels in the eye is likely to require either repeated administration of synthetic short interfering RNAs or vector-mediated expression of short hairpin RNAs. Further concerns that will need to be addressed include the possibilities of off-target non-specific responses and of a significant interferon response in vivo.

\section{Generic gene therapy strategies}

Although gene replacement and gene silencing strategies offer exciting prospects for the treatment of specific inherited retinal disorders, other disorders may be less amenable to these corrective approaches. These conditions include, in particular, those associated with abnormal retinal development and those in which retinal degeneration is advanced at birth. Furthermore, the development of individualized corrective gene therapy strategies for patients with disorders due to very rare mutations may be unfeasible. However, generic gene therapy strategies that aim not to correct the gene defect but to ameliorate its consequences offer the possibility of therapies that are widely applicable across a range of conditions. These approaches include the local expression of neurotrophic and anti-apoptotic proteins with the aim of promoting cell survival. rAAV-mediated gene delivery of neurotrophic factors is reported to prolong photoreceptor survival in experimental models of retinal degeneration. Such factors include fibroblast-growth factor (FGF), ciliary neurotrophic factor (CNTF) and glial-derived neurotrophic factor (GDNF). Vector-mediated CNTF-induced preservation of photoreceptors, however, appears to be associated with a paradoxical adverse effect on electroretinographic responses, an effect that may be a result of photoreceptor de-differentiation. ${ }^{43}$ The dose dependence and potential reversibility of this effect, and its implications for visual function in the long term are yet to be defined. In contrast, GDNF-induced rescue is accompanied by a significant improvement in retinal function. ${ }^{44}$

Furthermore, expression of GDNF in combination with gene replacement therapy augments the morphological and functional improvement mediated by gene replacement alone. ${ }^{44}$

\section{Challenges for gene therapy of inherited retinal disease}

Gene therapy is likely to be most successful when stable expression of the therapeutic transgene can be achieved at an appropriate level in diseases in which retinal development is unaffected and significant numbers of target cells survive at the point of intervention. In considering which conditions might be most amenable, a detailed understanding of their molecular and cellular pathology will be critical, including the relevance of developmental abnormalities and ambylopia.

Data from the clinical trials show that exposure of even a very small area of retina to vector can improve its function locally. It is not known at present whether there is any effect on the rate of retinal degeneration, nor whether transduction of a critical proportion of the retina is required to achieve this. Although preclinical studies suggest that long-term expression can be achieved by a single local administration of vector, the duration of expression in the human eye will not be known for many years. If the duration of expression is limited, further studies will be required to investigate the safety and efficacy of repeated administration.

The choice of specific retinal degenerations to be investigated in future clinical trials of gene therapy will be influenced by a number of considerations. These include the availability of relevant animal models for 
preclinical development, the disease prevalence, and the development of screening strategies to identify subjects for inclusion. Conditions in which gene therapy has the potential to improve visual function offer the prospect of a relatively rapid outcome, whereas those in which a progressive degeneration is delayed may require long-term assessment before a treatment effect can be expected. The potential to target certain conditions is currently limited by the capacity of available vector systems to package genomes. Although large genes can be delivered efficiently to the RPE using lentiviral vectors, delivery of large genes (such as ABCA4 in Stargardt's disease) to photoreceptor cells is more difficult to achieve. Recent progress suggests that this challenge can be addressed by improvements in the capacity of rAAV vectors ${ }^{18}$ or in photoreceptor targeting by lentiviral vectors. ${ }^{22}$

Although it is important that results of the first clinical trials are interpreted in an appropriately balanced fashion without raising expectations disproportionately, the first findings certainly offer hope to many thousands of individuals with inherited blindness for which no treatment is currently available.

\section{Acknowledgements}

James Bainbridge is a Wellcome Advanced Fellow (Grant $074617 / \mathrm{Z} / 04 / \mathrm{Z}$ ) and is supported by the NIHR Biomedical Research Centre for Ophthalmology at UCL and Moorfields Eye Hospital, London, UK.

\section{References}

1 Bessant DA, Ali RR, Bhattacharya SS. Molecular genetics and prospects for therapy of the inherited retinal dystrophies. Curr Opin Genet Dev 2001; 11(3): 307-316.

2 Andrieu-Soler C, Bejjani RA, de Bizemont T, Normand N, BenEzra D, Behar-Cohen F. Ocular gene therapy: a review of nonviral strategies. Mol Vis 2006; 12: 1334-1347.

3 Bejjani RA, Andrieu C, Bloquel C, Berdugo M, BenEzra D, Behar-Cohen F. Electrically assisted ocular gene therapy. Surv Ophthalmol 2007; 52(2): 196-208.

4 Bennett J, Wilson J, Sun D, Forbes B, Maguire A. Adenovirus vector-mediated in vivo gene transfer into adult murine retina. Invest Ophthalmol Vis Sci 1994; 35(5): 2535-2542.

5 Ali RR, Reichel MB, Byrnes AP, Stephens CJ, Thrasher AJ, Baker D et al. Co-injection of adenovirus expressing CTLA4-Ig prolongs adenovirally mediated lacZ reporter gene expression in the mouse retina. Gene Ther 1998; 5(11): 1561-1565.

6 Reichel MB, Ali RR, Thrasher AJ, Hunt DM, Bhattacharya SS, Baker D. Immune responses limit adenovirally mediated gene expression in the adult mouse eye. Gene Ther 1998; 5(8): 1038-1046.

7 Kumar-Singh R. Barriers for retinal gene therapy: separating fact from fiction. Vision Res 2008; 48(16): 1671-1680.
8 Ali RR, Reichel MB, Thrasher AJ, Levinsky RJ, Kinnon C, Kanuga $\mathrm{N}$ et al. Gene transfer into the mouse retina mediated by an adeno-associated viral vector. Hum $\mathrm{Mol}$ Genet 1996; 5(5): 591-594.

9 Flannery JG, Zolotukhin S, Vaquero MI, LaVail MM, Muzyczka N, Hauswirth WW. Efficient photoreceptor-targeted gene expression in vivo by recombinant adeno-associated virus. Proc Natl Acad Sci USA 1997; 94(13): 6916-6921.

10 Dreyer EB, Vorwerk CK, Zurakowski D, Simon PD, Bennett $\mathrm{J}$. Infection with adeno-associated virus may protect against excitotoxicity. Neuroreport 1999; 10(14): 2887-2890.

11 Weber M, Rabinowitz J, Provost N, Conrath H, Folliot S, Briot $\mathrm{D}$ et al. Recombinant adeno-associated virus serotype 4 mediates unique and exclusive long-term transduction of retinal pigmented epithelium in rat, dog, and nonhuman primate after subretinal delivery. Mol Ther 2003; 7(6): 774-781.

12 Surace EM, Auricchio A. Versatility of AAV vectors for retinal gene transfer. Vision Res 2008; 48(3): 353-359.

13 Lebherz C, Maguire A, Tang W, Bennett J, Wilson JM. Novel AAV serotypes for improved ocular gene transfer. J Gene Med 2008; 10(4): 375-382.

14 Buch PK, Bainbridge JW, Ali RR. AAV-mediated gene therapy for retinal disorders: from mouse to man. Gene Ther 2008; 15(11): 849-857.

15 Bainbridge JW, Mistry A, Schlichtenbrede FC, Smith A, Broderick C, De Alwis M et al. Stable rAAV-mediated transduction of rod and cone photoreceptors in the canine retina. Gene Ther 2003; 10(16): 1336-1344.

16 Acland GM, Aguirre GD, Bennett J, Aleman TS, Cideciyan AV, Bennicelli J et al. Long-term restoration of rod and cone vision by single dose rAAV-mediated gene transfer to the retina in a canine model of childhood blindness. Mol Ther 2005; 12(6): 1072-1082.

17 Min SH, Molday LL, Seeliger MW, Dinculescu A, Timmers AM, Janssen A et al. Prolonged recovery of retinal structure/function after gene therapy in an Rs1h-deficient mouse model of $\mathrm{x}$-linked juvenile retinoschisis. Mol Ther 2005; 12(4): 644-651.

18 Allocca M, Doria M, Petrillo M, Colella P, Garcia-Hoyos M, Gibbs D et al. Serotype-dependent packaging of large genes in adeno-associated viral vectors results in effective gene delivery in mice. J Clin Invest 2008; 118(5): 1955-1964.

19 Bainbridge JW, Stephens C, Parsley K, Demaison C, Halfyard A, Thrasher AJ et al. In vivo gene transfer to the mouse eye using an HIV-based lentiviral vector; efficient long-term transduction of corneal endothelium and retinal pigment epithelium. Gene Ther 2001; 8(21): 1665-1668.

20 Lotery AJ, Derksen TA, Russell SR, Mullins RF, Sauter S, Affatigato LM et al. Gene transfer to the nonhuman primate retina with recombinant feline immunodeficiency virus vectors. Hum Gene Ther 2002; 13(6): 689-696.

21 Balaggan KS, Binley K, Esapa M, Iqball S, Askham Z, Kan O et al. Stable and efficient intraocular gene transfer using pseudotyped EIAV lentiviral vectors. J Gene Med 2006; 8(3): 275-285.

22 Kong J, Kim SR, Binley K, Pata I, Doi K, Mannik J et al. Correction of the disease phenotype in the mouse model of Stargardt disease by lentiviral gene therapy. Gene Ther 2008; 15(19): 1311-1320.

23 Kostic C, Chiodini F, Salmon P, Wiznerowicz M, Deglon N, Hornfeld D et al. Activity analysis of housekeeping promoters using self-inactivating lentiviral vector delivery into the mouse retina. Gene Ther 2003; 10(9): 818-821. 
24 Gruter O, Kostic C, Crippa SV, Perez MT, Zografos L, Schorderet DF et al. Lentiviral vector-mediated gene transfer in adult mouse photoreceptors is impaired by the presence of a physical barrier. Gene Ther 2005; 12(11): 942-947.

25 Ali RR, Sarra GM, Stephens C, Alwis MD, Bainbridge JW, Munro PM et al. Restoration of photoreceptor ultrastructure and function in retinal degeneration slow mice by gene therapy. Nat Genet 2000; 25(3): 306-310.

26 Sarra GM, Stephens C, de Alwis M, Bainbridge JW, Smith AJ, Thrasher AJ et al. Gene replacement therapy in the retinal degeneration slow (rds) mouse: the effect on retinal degeneration following partial transduction of the retina. Hum Mol Genet 2001; 10(21): 2353-2361.

27 Pawlyk BS, Smith AJ, Buch PK, Adamian M, Hong DH, Sandberg MA et al. Gene replacement therapy rescues photoreceptor degeneration in a murine model of Leber congenital amaurosis lacking RPGRIP. Invest Ophthalmol Vis Sci 2005; 46(9): 3039-3045.

28 Zeng Y, Takada Y, Kjellstrom S, Hiriyanna K, Tanikawa A, Wawrousek E et al. RS-1 gene delivery to an adult Rs1 $\mathrm{h}$ knockout mouse model restores ERG b-wave with reversal of the electronegative waveform of $X$-linked retinoschisis. Invest Ophthalmol Vis Sci 2004; 45(9): 3279-3285.

29 Kjellstrom S, Bush RA, Zeng Y, Takada Y, Sieving PA. Retinoschisin gene therapy and natural history in the Rs1h-KO mouse: long-term rescue from retinal degeneration. Invest Ophthalmol Vis Sci 2007; 48(8): 3837-3845.

30 Alexander JJ, Hauswirth WW. Prospects for retinal conetargeted gene therapy. Drug News Perspect 2008; 21(5): 267-271.

31 Smith AJ, Schlichtenbrede FC, Tschernutter M, Bainbridge JW, Thrasher AJ, Ali RR. AAV-mediated gene transfer slows photoreceptor loss in the RCS rat model of retinitis pigmentosa. Mol Ther 2003; 8(2): 188-195.

32 Tschernutter M, Schlichtenbrede FC, Howe S, Balaggan KS, Munro PM, Bainbridge JW et al. Long-term preservation of retinal function in the RCS rat model of retinitis pigmentosa following lentivirus-mediated gene therapy. Gene Ther 2005; 12(8): 694-701.

33 Surace EM, Domenici L, Cortese K, Cotugno G, Di Vicino U, Venturi $\mathrm{C}$ et al. Amelioration of both functional and morphological abnormalities in the retina of a mouse model of ocular albinism following AAV-mediated gene transfer. Mol Ther 2005; 12(4): 652-658.

34 Lorenz B, Wabbels B, Wegscheider E, Hamel CP, Drexler W, Preising MN. Lack of fundus autofluorescence to 488 nanometers from childhood on in patients with early-onset severe retinal dystrophy associated with mutations in RPE65. Ophthalmology 2004; 111(8): 1585-1594.
35 Narfstrom K, Katz ML, Bragadottir R, Seeliger M, Boulanger A, Redmond TM et al. Functional and structural recovery of the retina after gene therapy in the RPE65 null mutation dog. Invest Ophthalmol Vis Sci 2003; 44(4): 1663-1672.

36 Le Meur G, Stieger K, Smith AJ, Weber M, Deschamps JY, Nivard D et al. Restoration of vision in RPE65-deficient Briard dogs using an AAV serotype 4 vector that specifically targets the retinal pigmented epithelium. Gene Ther 2007; 14(4): 292-303.

37 Bainbridge JW, Smith AJ, Barker SS, Robbie S, Henderson R, Balaggan K et al. Effect of gene therapy on visual function in Leber's congenital amaurosis. N Engl J Med 2008; 358(21): 2231-2239.

38 Maguire AM, Simonelli F, Pierce EA, Pugh Jr EN, Mingozzi F, Bennicelli J et al. Safety and efficacy of gene transfer for Leber's congenital amaurosis. $N$ Engl J Med 2008; 358(21): 2240-2248.

39 Cideciyan AV, Aleman TS, Boye SL, Schwartz SB, Kaushal S, Roman AJ et al. Human gene therapy for RPE65 isomerase deficiency activates the retinoid cycle of vision but with slow rod kinetics. Proc Natl Acad Sci USA 2008; 105(39): 15112-15117.

40 Hauswirth W, Aleman TS, Kaushal S, Cideciyan AV, Schwartz SB, Wang L et al. Phase I trial of Leber congenital amaurosis due to RPE65 mutations by ocular subretinal injection of adeno-associated virus gene vector: short-term results. Hum Gene Ther 2008 [e-pub ahead of print].

41 Gorbatyuk MS, Pang JJ, Thomas Jr J, Hauswirth WW, Lewin AS. Knockdown of wild-type mouse rhodopsin using an AAV vectored ribozyme as part of an RNA replacement approach. Mol Vis 2005; 11: 648-656.

42 O'Reilly M, Palfi A, Chadderton N, Millington-Ward S, Ader M, Cronin T et al. RNA interference-mediated suppression and replacement of human rhodopsin in vivo. Am J Hum Genet 2007; 81(1): 127-135.

43 Schlichtenbrede FC, MacNeil A, Bainbridge JW, Tschernutter M, Thrasher AJ, Smith AJ et al. Intraocular gene delivery of ciliary neurotrophic factor results in significant loss of retinal function in normal mice and in the Prph2Rd2/Rd2 model of retinal degeneration. Gene Ther 2003; 10(6): 523-527.

44 Buch PK, MacLaren RE, Duran Y, Balaggan KS, MacNeil A, Schlichtenbrede FC et al. In contrast to AAV-mediated Cntf expression, AAV-mediated Gdnf expression enhances gene replacement therapy in rodent models of retinal degeneration. Mol Ther 2006; 14(5): 700-709. 